\title{
Exercise testing without interruption of medication for refining the selection of mildly symptomatic patients for prognostic coronary angiography
}

Richard Lim, Imad Kreidieh, Lorraine Dyke, Janice Thomas, Duncan S Dymond

\begin{abstract}
Objective-To examine how exercise testing on background medical treatment affects the ability of the test to predict prognostically important patterns of coronary anatomy in patients with a high clinical probability of coronary artery disease but who are well controlled on medication.

Design-Prospective study.

Setting-Regional cardiothoracic centre and referring district general hospital.

Patients-84 patients with a history of typical angina or definite myocardial infarction and mild symptoms who had been placed on the waiting list for prognostic angiography.

Intervention-Maximal exercise electrocardiography and radionuclide ventriculography performed off and on medication, followed by angiography within three months.
\end{abstract}

Main outcome measure-Prognostically important coronary artery disease for which early surgery might be recommended purely on prognostic grounds, irrespective of symptoms.

Results-Coronary artery disease was present in 71/84 (85\%) patients; in 28/84 (33\%) patients this was prognostically important. When the result was strongly positive, the predictive accuracy for prognostically important disease was 0.46 off and 0.62 on medication for the exercise electrocardiogram and 0.71 off and 0.82 on medication for exercise radionuclide ventriculography. The likelihood ratio was 1.00 off and 1.36 on medication for exercise electrocardiography and 2.54 off and 10.5 on medication for exercise radionuclide ventriculography. In stepwise logistic regression, the test identified as the strongest predictor of prognostically important disease was exercise radionuclide ventriculography on medication for which the improvement $\chi^{2}$ was 28 (p <0.0001). With the regression model, the probability of important disease is $92 \%$ if exercise radionuclide ventriculography on medication is at least strongly positive, compared with $16 \%$ if the result is normal or just positive.

Conclusion-In patients likely to have coronary disease, exercise testing should be performed without interruption of medication to optimise its ability to identify those with prognostically important disease, and to help to avoid unnecessary or premature angiography in those who are well controlled on medical treatment.

(Br Heart f 1994;71:334-340)

In patients with a high clinical probability of coronary artery disease, a negative or positive exercise test performed conventionally may not appreciably alter the odds of disease from those before the test, ${ }^{1-3}$ but if strongly positive despite medication, the test may increase the odds of disease and have prognostic significance. In such patients, the main role of exercise testing is to select for invasive coronary angiography those who, although adequately controlled by medication, may none the less benefit from having their coronary anatomy defined for potential bypass surgery on prognostic grounds. This prospective study was therefore undertaken in mildly symptomatic patients to see how performing the test on background medical treatment affects the ability of exercise electrocardiography and radionuclide ventriculography to predict prognostically important patterns of coronary anatomy.

Patients and methods

PATIENTS

The study population consisted of 84 consecutive unrevascularised patients with a history of classical angina or a previous definite myocardial infarction ( $n=24)$ who had been placed on the waiting list for prognostic coronary angiography. The median (range) age was 61 (39-75) years and 79 were men. Thirty one patients $(37 \%)$ had been referred to our unit directly by their general practitioner and 53 by a hospital consultant. Informed consent was obtained from all patients.

\section{SYMPTOMS}

Patients were excluded from the study if they had been referred primarily because of intractable symptoms or recent unstable angina. Our patients were the initial cohort being screened for a multicentre trial on silent ischaemia that required participants to be adequately controlled (angina class $\leqslant$ II) on conventional anti-ischaemic medication. ${ }^{4}$ In the four weeks before screening, 20 patients 
were in angina class II, 41 were in angina class I, and $23(27 \%)$ were asymptomatic.

BACKGROUND ANTI-ISCHAEMIC MEDICATION Fifty one patients $(61 \%)$ were taking $\beta$-blockers, 45 were taking calcium antagonists, and 29 were on long acting nitrates. Forty eight patients $(57 \%)$ were taking one drug, 31 were taking two drugs, and only five were receiving three.

\section{EXERCISE TESTING}

Patients were excluded if they had atrial fibrillation, significant valvar disease, heart failure, or electrocardiographic ST segments that would be uninterpretable during exercise testing-for example, left bundle branch block. Each patient underwent maximal symptom limited exercise, electrocardiography and radionuclide ventriculography after all antiischaemic medication (except aspirin) had been stopped for four days. These were performed on the same day with a rest period of 90 minutes between tests. Results of exercise electrocardiography and radionuclide ventriculography were interpreted independently of each other by two experienced observers.

Two weeks after the resumption of usual medication, maximal symptom limited exercise electrocardiography and radionuclide ventriculography were repeated if the respective baseline test off medication was positive. Again the results of electrocardiography and radionuclide ventriculography were analysed independently of each other by the same two experienced observers blinded to the results of the initial tests.

\section{Exercise electrocardiography}

The method specified by the study of silent ischaemia was the modified Balke treadmill protocol. ${ }^{4}$ Compared with the Bruce protocol, which is characterised by uneven stepwise increases in workload, the gentler Balke protocol allows an almost linear increase in oxygen consumption at 1.5 metabolic equivalents of the task (METs) increments between stages, achieving a maximum workload of 15.1 METs. This permits cleaner 12 lead electrocardiographic traces, facilitates the identification of the onset of $0.1 \mathrm{mV}(1 \mathrm{~mm})$ of ST segment depression and the measurement of its maximum amplitude, and may be more reliable for studying the effects of intervention. ${ }^{56}$

\section{Exercise radionuclide ventriculography}

Left ventricular ejection fraction (LVEF) was measured at rest and peak upright cycle exercise by the first pass method with the Scinticor multicrystal gamma camera system (Scinticor Inc, Wisconsin, USA), as previously described. ${ }^{7}$

\section{POSITIVITY CRITERIA FOR ISCHAEMIA}

Although other test variables-for example, angina, ${ }^{8}$ exercise capacity, ${ }^{9}$ and peak exercise LVEF $^{10}$-may suggest significant disease, electrocardiographic ST segment depres- sion, ${ }^{11}$ and the response of LVEF to exercise $^{12,13}$ as the objective indices of inducible ischaemia most widely accepted and adopted in clinical practice, were regarded as the quantitative indices of primary interest. In this study, the following predefined criteria were applied.

\section{Exercise electrocardiography}

(a) Positive-Downsloping, planar, or upsloping ST segment depression $\geqslant 1 \mathrm{~mm}$ $(0 \cdot 1 \mathrm{mV})$ at $80 \mathrm{~ms}$ from the J point.

(b) Strongly positive-Downsloping or planar ST segment depression $\geqslant 2 \mathrm{~mm}$ at $80 \mathrm{~ms}$ from the J point.

(c) Very strongly positive-Downsloping or planar ST segment depression $\geqslant 2 \mathrm{~mm}$ occurring at low workload - that is, within stage 4 ( $<6 \mathrm{~min},<6 \mathrm{METs}$ ) of the exercise protocol.

\section{Exercise radionuclide ventriculography}

(a) Positive-Fall of $\geqslant 1 \%$ in LVEF from rest to exercise plus an exercise LVEF $<50 \%$.

(b) Strongly positive-Fall of $>5 \%$ in LVEF from rest to exercise plus an exercise LVEF $<45 \%$.

(c) Very strongly positive-Fall of $>10 \%$ in LVEF from rest to exercise plus an exercise LVEF $<45 \%$.

\section{CORONARY ANGIOGRAPHY}

This was performed in multiple projections with the Judkin's method within three months after the first exercise tests. Angiograms were interpreted by the patients' consultant without knowledge of the paired exercise test results.

Significant coronary artery disease was defined visually as $\geqslant 50 \%$ stenosis in luminal diameter in at least one major epicardial artery. A stenosis was considered to be proximal if located in the left anterior descending artery (LAD) before the first septal or diagonal branch; the left circumflex artery before the first marginal branch; or the proximal third of the right coronary artery.

Prognostically important coronary artery disease-the study endpoint-was defined as disease for which early bypass surgery might be considered purely on prognostic grounds, ${ }^{14-16}$ irrespective of symptoms. This term comprised the following patterns of coronary anatomy: stenosis of the left main stem $\geqslant 50 \%$ with or without disease elsewhere; proximal three vessel disease; three vessel disease including the proximal LAD; proximal 2 vessel disease including the $\mathrm{LAD}$; or 2 vessel disease including the proximal LAD.

\section{STATISTICS}

The likelihood ratio (odds after/before test) gives an indication of the value of a test result for increasing the certainty of prognostically important coronary artery disease: a higher ratio implies a more useful test.

To determine which test was most predictive of prognostically important coronary artery disease, logistic regression analysis (SPSS/PC + version $4 \cdot 0$ ) was also performed. 
Table 1 Baseline exercise testing off medication

\begin{tabular}{lll}
\hline & \multicolumn{2}{l}{ No of patients } \\
\cline { 2 - 3 } & $\begin{array}{l}\text { Electrocardio- } \\
\text { graphy } \\
(\%)\end{array}$ & $\begin{array}{l}\text { Radionuclide } \\
\text { ventriculography } \\
(\%)\end{array}$ \\
\hline Negative & $7(8)$ & $36(43)$ \\
Positive & $20(24)$ & $16(19)$ \\
Strongly positive & $41(49)$ & $8(10)$ \\
Very strongly positive & $16(19)$ & $24(29)$ \\
\hline
\end{tabular}

Table 2 Patterns of coronary anatomy

\begin{tabular}{lr}
\hline Coronary artery disease & No \\
\hline Prognostically important: & \\
Left main stem & 4 \\
Proximal 3 VD or 3 VD including proximal LAD & 12 \\
Proximal 2 VD including LAD & 6 \\
2 VD including proximal LAD & 6 \\
Other: & 8 \\
Other 3 VD & 9 \\
Other 2 VD & 11 \\
Isolated proximal LAD & 15 \\
Other 1 VD & 13 \\
No significant stenosis
\end{tabular}

$\mathrm{LAD}$, left anterior descending artery; $\mathrm{VD}$, vessel disease.

The variables considered for entry into the model in a forward stepwise fashion were: exercise electrocardiography off medication, exercise electrocardiography on medication, exercise radionuclide ventriculography off medication, and exercise radionuclide ventriculography while taking medication. A test result on medication was assumed to be normal and was coded accordingly if the baseline test off medication was normal.

\section{Results}

BASELINE EXERCISE TESTING OFF MEDICATION With the predetermined positivity criteria, only seven patients (8\%) had a negative exercise electrocardiography at baseline compared with 36 patients (43\%) with a negative exercise radionuclide ventriculography (table 1 ).
CORONARY ANGIOGRAPHY

Table 2 shows the breakdown of angiographic results. In this population, the prevalence of significant coronary artery disease was $71 / 84$ $(85 \%)$ patients. Four patients $(4 \cdot 8 \%)$ had significant stenosis of the left main stem. The overall prevalence of prognostically important coronary artery disease was 28/84 (33\%) patients.

\section{PREDICTIVE VALUES AND ACCURACY}

Exercise electrocardiography

The mean amplitude of ST segment depression was reduced significantly, and to a similar degree, by medication in patients both with and without prognostically important coronary artery disease (table 3). Patients with prognostically important coronary artery disease exercised less than those without, whether off or on medication. Medication improved duration of exercise during testing only in patients with prognostically important coronary artery disease.

Overall, performing the exercise electrocardiogram on medication did not impair the test's predictive values and accuracy; indeed, with criteria for at least a strongly positive result, there was a trend towards improvement in test performance (table 4).

\section{Exercise radionuclide ventriculography}

Patients with prognostically important coronary artery disease had a lower mean exercise LVEF and a greater mean exercise induced fall in LVEF during testing both off and on medication than did patients without prognostically important coronary artery disease (table 5). Further, in patients with prognostically important coronary artery disease these indices did not improve during testing on medication by contrast with those without prognostically important coronary artery disease.

Testing on medication significantly

Table 3 Exercise electrocardiographic variables off and on medication

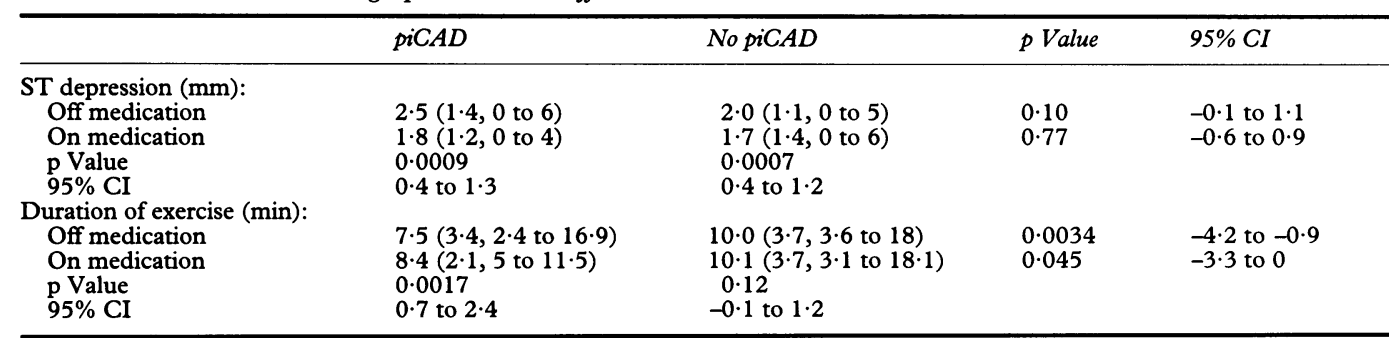

Values are means (SD, range); 95\% CI, 95\% confidence interval for difference between means; piCAD, prognostically important coronary artery disease.

Table 4 Test performance of exercise electrocardiography

\begin{tabular}{|c|c|c|c|c|c|}
\hline & Sensitivity (\%) & Specificity (\%) & $P P V(\%)$ & $N P V(\%)$ & $P A(\%)$ \\
\hline \multicolumn{6}{|l|}{ Strongly positive: } \\
\hline Off medication & 62 & 38 & 31 & 69 & 46 \\
\hline On medication & & & & & \\
\hline McNemar's or $\chi^{2}$ test & $z=0.87$ & $z=2.08$ & $\chi^{2}=0.32$ & $\chi^{2}=0.08$ & $z=1 \cdot 17$ \\
\hline \multirow{2}{*}{\multicolumn{6}{|c|}{ Very strongly positive: }} \\
\hline & & & & & \\
\hline On medication & $\begin{array}{r}29 \\
5\end{array}$ & $\begin{array}{l}87 \\
98\end{array}$ & $\begin{array}{l}50 \\
50\end{array}$ & $\begin{array}{l}73 \\
70\end{array}$ & $\begin{array}{l}69 \\
69\end{array}$ \\
\hline McNemar's or $\chi^{2}$ test & $z=1.51$ & $z=0.43$ & $\chi^{2}=0$ & $\chi^{2}=0.18$ & $z=0.1$ \\
\hline p Value & NS & NS & NS & NS & NS \\
\hline
\end{tabular}

PPV, positive predictive value; NPV, negative predictive value; PA, predictive accuracy. 
Table 5 Variables of exercise radionuclide ventriculography off and on medication

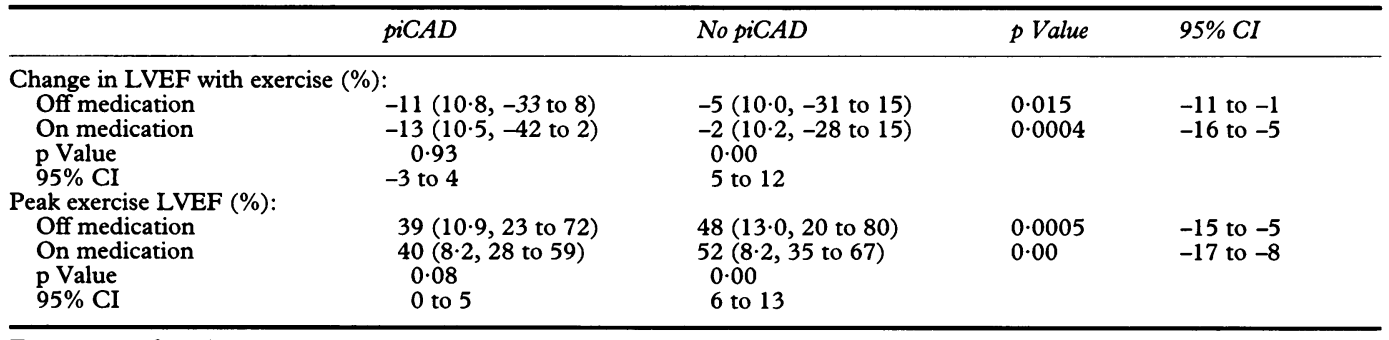

Footnotes as for table 3.

Table 6 Test performance of exercise radionuclide ventriculography

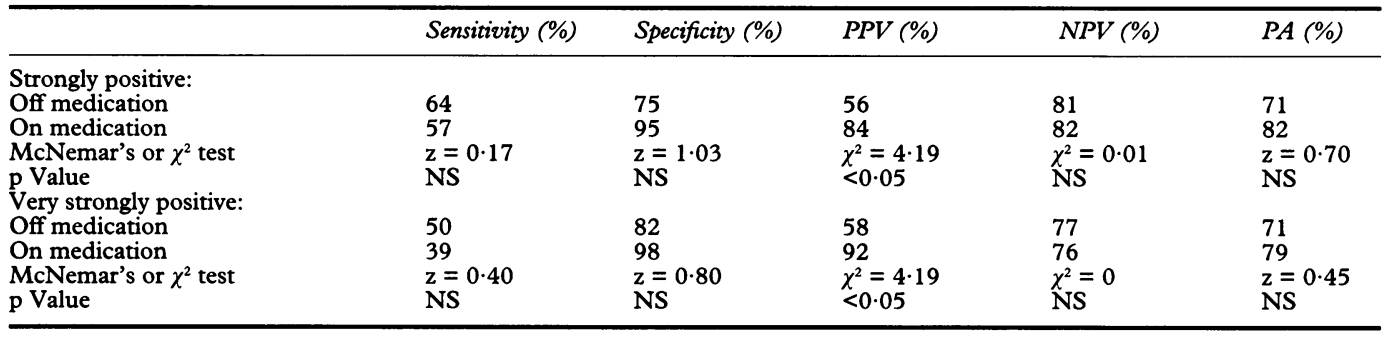

Abbreviations as for table 4

improved the positive predictive value of a strongly or very strongly positive result, without impairing the negative predictive value (table 6). Overall, there was a clear trend towards improvement in predictive accuracy by testing on medication.

\section{LIKELIHOOD RATIO}

Exercise electrocardiography

The likelihood ratio for a strongly positive result from exercise electrocardiography off medication was $1 \cdot 00$, - that is, such a test result did not alter the likelihood of prognostically important coronary artery disease at all (table 7). A similar result on medication, however, increased the likelihood of prognostically important coronary artery disease by $36 \%$ (figure). When the result was very strongly positive, the likelihood of prognostically important coronary artery disease was increased by over twofold, irrespective of whether the test was done off or on medication.

Exercise radionuclide ventriculography

The likelihood of prognostically important coronary artery disease was increased by 2.54 times if an exercise radionuclide ventriculography was strongly positive off medication, but a similar result on medication further improved the certainty by over fourfold (table

Table 7 Likelihood ratio (LR)

\begin{tabular}{lcc}
\hline & Odds after test & $L R$ \\
\hline \multicolumn{2}{c}{ Exercise electrocardiography } \\
Strongly positive: & 0.45 & \\
Off medication & 0.61 & 1.00 \\
On medication & 1.00 & 1.36 \\
Very strongly positive: & 1.00 & 2.22 \\
$\quad$ Off medication & 2.22 \\
On medication & Exercise radionuclide ventriculography & \\
Strongly positive: & 1.27 & 2.54 \\
Off medication & 5.25 & 10.5 \\
On medication & 1.38 & 2.76 \\
Very strongly positive: & 11.5 & 23.0 \\
$\quad$ Off medication & & \\
On medication & &
\end{tabular}

7; figure). A strongly positive result when on medication increased the certainty of prognostically important coronary artery disease by 10 -fold, and a very strongly positive result by 23-fold (table 7). When off medication, there was little difference in likelihood ratio between a strongly positive result and a very strongly positive result. When the result was very strongly positive, the incremental value of testing on medication over testing off medication for increasing the certainty of important disease was eightfold (figure).

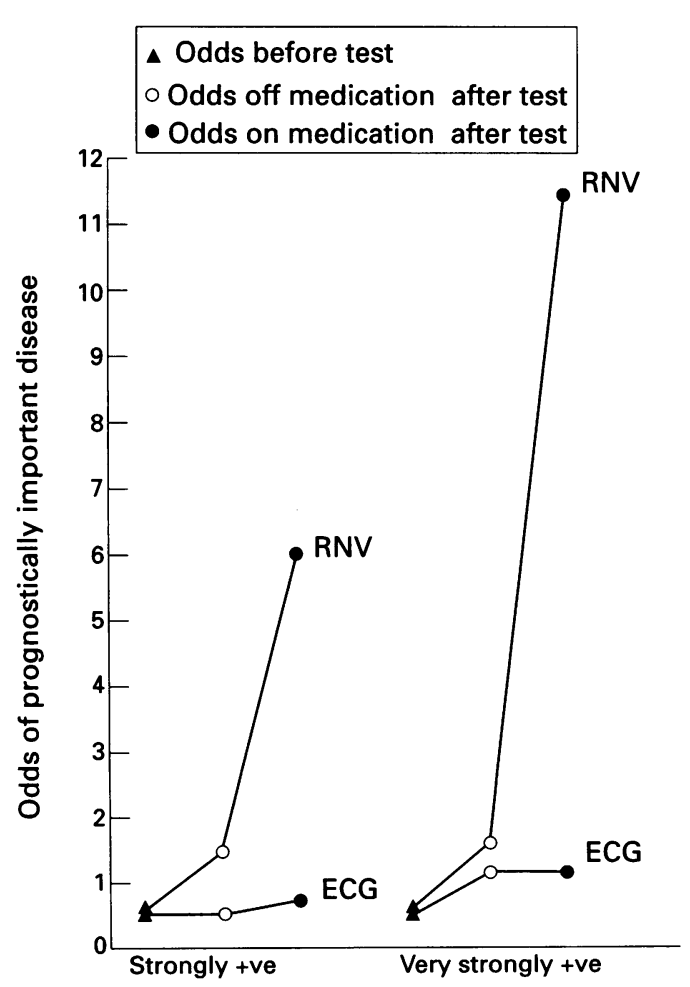

Effect of results of exercise electrocardiography (ECG) and radionuclide ventriculography (RNV) on and off medication on odds of presence of prognostically important disease. 
Table 8 Variables considered in logistic regression analysis

\begin{tabular}{|c|c|c|c|c|}
\hline \multirow[b]{2}{*}{ Variable } & \multicolumn{2}{|l|}{ Step 1} & \multicolumn{2}{|l|}{ Step 2} \\
\hline & Unadjusted $\chi^{2}$ & p Value & Adjusted $\chi^{2}$ & $p$ Value \\
\hline \multicolumn{5}{|c|}{ Exercise electrocardiography: } \\
\hline Off medication & 0.00 & 0.987 & 0.00 & 0.971 \\
\hline On medication & $0 \cdot 74$ & 0.389 & $0 \cdot 33$ & 0.565 \\
\hline \multicolumn{5}{|c|}{ Exercise radionuclide ventriculography: } \\
\hline Off medication & $14 \cdot 18$ & 0.0002 & $1 \cdot 78$ & $0 \cdot 182$ \\
\hline On medication & $28 \cdot 41$ & 0.0000 & $27 \cdot 99$ & 0.0000 \\
\hline
\end{tabular}

\section{LOGISTIC REGRESSION}

The first variable chosen was exercise radionuclide ventriculography on medication. The improvement $\chi^{2}$ was $28(\mathrm{p}<0.0001)$ with a goodness of fit of $68(p=0 \cdot 41)$. After controlling for exercise radionuclide ventriculography on medication, no other variables were identified as significant independent contributors to the prediction of prognostically important coronary artery disease (table 8). The final logistic regression model was $\log _{e}(p / 1-p)$ $=0.427-2.058$ RNVon where $\mathrm{p}=$ probability of prognostically important coronary artery disease, $(p / 1-p)=$ odds, the SE of each coefficient $=0.55$, and RNVon (exercise radionuclide ventriculography on medication) $=1$ for a normal/positive result, or -1 for a strongly positive/very strongly positive result.

With the model, the probability of prognostically important coronary artery disease is $92 \%$ if the exercise radionuclide ventriculogram on medication gives at least a strongly positive result, compared with $16 \%$ if the result is normal or just positive.

\section{Discussion}

\section{STUDY RATIONALE}

Faced with a patient with a history that indicates ischaemic heart disease but who has few ongoing symptoms, what is the mandate for invasive coronary angiography? Ischaemia that is silent may still be evident even in patients rendered asymptomatic by antianginal medication, ${ }^{17}$ so many deserve objective assessment.

The exercise test is the most common initial method, but wide variation in practice over stopping medication for the test has been highlighted recently. ${ }^{18} 19$ Just as its predictive performance is influenced crucially by the prevalence of disease, ${ }^{2}$ its interpretation depends on the purpose of testing, ${ }^{20}$ as does arguably the issue of whether it should be performed off or on medication. ${ }^{21}$ When there is clinically already a strong likelihood of underlying coronary artery disease, the usefulness of exercise testing shifts to its ability to assess prognosis. $^{22}$ In such patients, rather than merely to confirm the presence of disease, exercise testing has potentially greater clinical value if it can predict angiographic patterns of disease for which early surgery might be recommended on prognostic grounds. By testing when on medication, can the tests be refined for the cost-conscious management of patients who are mildly symptomatic yet have a high likelihood of coronary artery disease?

In such a population, various strategies are available. The most conservative is to offer invasive coronary angiography only if and when symptoms become troublesome; such an expectant policy is less than satisfactory in as much as it is based only on the subjective assessment of symptoms. The aggressive approach is to catheterise everyone who may potentially benefit from revascularisation on prognostic grounds. Although this approach might be ideal were angiographic data to be complemented by functional testing for full prognostic assessment, it has considerable resource implications for any constrained health service. Between these two extreme policies is the strategy of selecting for timely angiography those patients with a prognostically bad exercise test.

\section{LIMITATIONS OF PREVIOUS STUDIES}

This prospective study differs from previous work in several respects. Specifically targeting patients who were mildly symptomatic yet had a high likelihood of coronary artery disease, it assessed the impact of background medication on the value of exercise tests in a population who were already referred for coronary angiography. This avoids referral bias whereby positive test responders are preferentially referred for angiography. In one study, only 24 of 57 patients actually underwent angiography "at the discretion of physicians involved in the management of individual patients". ${ }^{23}$ Such bias from performing angiography only in selected cases was compounded by inappropriate comparison of the results of a single exercise test in patients actually taking $\beta$ blockers with a group not prescribed $\beta$ blockers. ${ }^{24} 25$

With a single dose of $\beta$ blocker, Marcomichelakis et al found improved specificity and predictive value without loss of sensitivity for detecting any coronary artery disease. ${ }^{26}$ Mukharii et al concluded that antianginal treatment reduced the value of exercise electrocardiography as a screening tool for three vessel or left main stem disease after examining the effect of trial drugs (calcium blockers and nitrates) with the endpoint of only $1 \mathrm{~mm}$ maximum ST segment depression. ${ }^{23}$ Ho et al reported that background $\beta$ blockade impaired the ability of exercise electrocardiography to identify subjects already documented to have extensive coronary artery disease. ${ }^{27}$ All these workers used only two or three electrocardiographic leads to monitor the ST segment.

Finally, studies comparing exercise radionuclide ventriculography with exercise electrocardiography have not examined systematically the effect of medication on test performance. ${ }^{28-30}$ Indeed, in some, comparison was only indirect ${ }^{24}{ }^{31}$ or medication details were omitted. ${ }^{32}$ There may also have been some degree of investigator bias against exercise electrocardiography ${ }^{33}$; for instance, when the study of Borer et al was conducted, only two leads were used to monitor the electrocardiogram. ${ }^{28}$ 
disease is high, the likelihood ratio of one for strongly positive exercise electrocardiography off medication clearly implies that such a result has no value for increasing the certainty of prognostically important coronary artery disease beyond clinical assessment. This limited incremental value of exercise electrocardiography performed off medication to detect coronary anatomy needing surgery is disappointing given that it is such an established, widely practised, and relatively inexpensive test. Testing on medication, however, was more useful if it produced a strongly positive result, and even more so if very strongly positive.

For exercise radionuclide ventriculography, prognostic testing should definitely be done on medication to optimise predictive performance. The superior performance of exercise radionuclide ventriculography on medication in both Bayesian and logistic regression analyses makes it the test of choice where it is available. Applying the logistic regression model, the probability of prognostically important disease is over $90 \%$ if the result is at least strongly positive. With a normal or just positive result, the probability of important disease falls to $16 \%$. In any individual patient, the level of probability required to cross comfortably the decision threshold for angiography remains a matter of clinical judgment. Furthermore, functional testing cannot identify every single case of important coronary anatomy. There is, however, prognostic heterogeneity even in triple vessel disease, and a satisfactory functional test result portends a satisfactory prognosis. ${ }^{34-36}$ Thus if prognostic indicators from non-invasive testing are good, there is arguably no urgency or need even for invasive coronary angiography.

Millane and Ward have suggested that "all patients should be exercised first off drugs to 'objectively assess symptoms and risk and then again when they are symptomatically stable on drugs. Patients should then be referred for further investigation on the basis of the first exercise test, with the urgency of referral being based on the second test". ${ }^{37}$ This places an unnecessary burden on limited resources, given that this study indicates that in the prognostic assessment of patients who are well controlled but none the less have a high clinical probability of disease, exercise testing should be undertaken only on medication, there being no additional role for testing off treatment. Conventional background antianginal medication certainly does not impair the ability of maximal exercise tests to predict prognostically important patterns of coronary artery disease. Indeed, the clinical value of the testparticularly exercise radionuclide ventriculography-is enhanced by performing it on medication.

\section{FUTURE DIRECTIONS}

The ultimate question is: can coronary revascularisation and therefore angiography be safely deferred in medically treated minimally symptomatic patients unless exercise test results on medical treatment are bad or, for those with initially satisfactory results, deteriorate? Should not patients with prognostically bad test results despite medication join those with troublesome symptoms despite medication in being potential candidates for intervention? Despite the widespread use of and reliance on exercise testing to guide the management of ischaemic heart disease, no definitive trial has been reported of patients randomised by exercise test result to either early angiography with a view to revascularisation, or initially continuing medical treatment. Because the issue is early intervention $v$ continuing with medication, not intervention $v$ no treatment, future trials will be more relevant if based on exercise test results on, not off, medication. ${ }^{21}$ The clinical and resource implications point to a need for a randomised multicentre study to refine and rationalise the selection of patients for invasive coronary angiography and revascularisation.

This study was supported by a project grant from the St Bartholomew's Hospital Joint Research Board. RL was funded under a National Institutes of Health research contract. We thank June Elstob, Amanda Powe, and Sarah Way for technical assistance with exercise electrocardiography.

1 Diamond GA, Forrester JS. Analysis of probability as an aid in the clinical diagnosis of coronary artery disease. $N$ aid in the clinical diagnosis of

2 Epstein SE. Implications of probability analysis on the strategy used for noninvasive detection of coronary artery disease. Role of single or combined use of exercise electrocardiographic testing, radionuclide cineangiography and myocardial perfusion imaging. $\mathrm{Am} \mathcal{f}$ Cardiol 1980;46:491-9.

3 Goldman L, Cook EF, Mitchell N, Flatley M, Sherman H, Rosati $\mathrm{R}$, et al. Incremental value of the exercise test for diagnosing the presence or absence of coronary artery disease. Circulation 1982;66:945-53.

4 The ACIP Investigators. Asymptomatic cardiac ischemia pilot study (ACIP). Am $\mathcal{F}$ Cardiol 1992;70:744-7.

5 Redwood DR, Rosing DR, Goldstein RE, Beiser GD, Epstein SE. Importance of the design of an exercise protocol in the evaluation of patients with angina pectoris. Circulation 1971;43:618-28.

6 Myers J, Froelicher VF. Optimizing the exercise test for pharmacological investigations. Circulation 1990;82: 1839-46.

7 Lim R, Dyke L, Thomas J, Dymond DS. Novel approach to the interpretation of long-term "deterioration" in ejecthe interpretation of long-term "deterioration" in ejection fraction in individual patients
disease. $\mathrm{Br} \mathrm{Heart} \mathcal{1}$ 1993;70:226-32.

8 Mark DB, Shaw L, Harrell FE Jr, Hlatky MA, Lee KL, Bengston JR, et al. Prognostic value of a treadmill exercise score in outpatients with suspected coronary artery disease. N Engl F Med 1991;325:849-53.

9 Morris CK, Ueshima K, Kawaguchi T, Hideg A, Froelicher VF. The prognostic value of exercise capacity: a review of the literature. Am Heart $f$ 1991; 122:1423-31.

10 Gibbons RJ. Rest and exercise radionuclide angiography for diagnosis in chronic ischemic heart disease. Circulation 1991;84(suppl I):I-93-9.

11 Chaitman BR. The changing role of the exercise electrocardiogram as a diagnostic and prognostic test for chronic ischemic heart disease. $f$ Am Coll Cardiol 1986;8:1195-210.

12 Jones RH, Floyd RD, Austin EH, Sabiston DC Jr. The role of radionuclide angiocardiography in the preoperative prediction of pain relief and prolonged survival foltive prediction of pain relief and prolonged survival following coronary

13 Wallis JB, Borer JS. Identification of "surgical" coronary anatomy by exercise radionuclide cineangiography. $A m \mathcal{F}$ Cardiol 1991;68:1150-7.

14 CASS Principal Investigators and their Associates. Coronary artery surgery study (CASS). A randomized trial of coronary artery bypass surgery. Survival data. Circulation 1983;68:939-50.

15 Detre KM, Takaro $T$, Hultgren $H$, Peduzzi $P$, and the Study Participants. Long-term mortality and morbidity results of the veterans administration randomized trial of coronary artery bypass surgery. Circulation 1985; 72(suppl V):V-84-9.

6 Varnauskas E, and the European Coronary Surgery Study Group. Twelve-year follow-up of survival in the randomized European coronary surgery study. $N$ Engl $\mathcal{F}$ Med 1988;319:332-7.

17 Mulcahy D, Keegan J, Lindsay D, Sparrow J, Park A, Wright C, et al. Silent myocardial ischaemia in patients referred for coronary bypass surgery because of angina: a 
comparison with patients whose symptoms were well controlled on medical treatment. Br Heart $\mathcal{f} 1989$, 61:496-501.

18 Underwood R, Gibson C, Tweddel A, Flint J on behalf of the British Nuclear Cardiology Group. A survey of nuclear cardiological practice in Great Britain. Br Heart $\mathcal{f}$ 1992;67:273-7

19 Muir KW, Rodger JC, DeBono JS, McDonald H Irving JB. Drugs and the exercise test. $B M \mathcal{F} 1992 ; 305$ 808-9.

20 Rozanski A, Diamond GA, Forrester JS, Berman DS, Morris D, Jones RH, et al. Should the intent of testin influence its interpretation? $\mathcal{F} \mathrm{Am}$ Coll Cardiol 1986; 7:17-24.

21 Lim R, Dymond DS. Should antianginal medication be stopped for exercise testing? Lancet 1992;340:161-2.

22 Morise AP, Detrano R, Bobbio M, Diamond GA Development and validation of a logistic regressionderived algorithm for estimating the incremental probability of coronary artery disease before and after exercise bility of coronary artery disease before and a

23 Mukharji J, Kremers M, Lipscomb K, Blomqvist CG. Early positive exercise test and extensive coronary disease: effect of antianginal therapy. $A m \mathcal{F}$ Cardiol 1985;55:267-70.

24 Jones RH, McEwan P, Newman GE, Port S, Rerych SK, Scholz PM, et al. Accuracy of diagnosis of coronary artery disease by radionuclide measurement of left ventricular function during rest and exercise. Circulation 1981;64:586-601.

25 Murray DP, Tan LB, Salih M, Weissberg P, Murray RG, Littler WA. Does $\beta$ adrenergic blockade influence the prognostic implications of post-myocardial infarction exercise testing? $B r$ Heart f 1988;60:474-9.

26 Marcomichelakis J, Donaldson R, Green J, Joseph S, Kelly HB, Taggart P, et al. Exercise testing after betablockade: improved specificity and predictive value in detecting coronary heart disease. Br Heart $\mathcal{f} 1980 ; 43$ : 252-61.

27 Ho SWC, McComish MJ, Taylor RR. Effect of beta adrenergic blockade on the results of exercise testing related to the extent of coronary artery disease. $A m \mathcal{J}$ Cardiol 1985;55:258-62.

28 Borer JS, Kent KM, Bacharach SL, Green MV, Rosing DR, Seides SF, et al. Sensitivity, specificity and predictive accuracy of radionuclide cineangiography during exercise in patients with coronary artery disease. Comparison with exercise electrocardiography.

29 Campos CT, Chu HW, D'Agostino HJ Jr, Jones RH. Comparison of rest and exercise radionuclide angiocardiography and exercise treadmill testing for diagnosis of anatomically extensive coronary artery disease. Circulation 1983;67:1204-10.

30 Bobbio M, Pollock BH, Cohen I, Diamond GA. Comparative accuracy of clinical tests for diagnosis and prognosis of coronary artery disease. $\mathrm{Am} \mathrm{f}$ Cardiol 1988;62:896-900

31 Gibbons RJ, Fyke FE, Clements IP, Lapeyre AC, Zinsmeister AR, Brown ML. Noninvasive identification of severe coronary artery disease using exercise radionuclide angiography. $\varsubsetneqq \mathrm{Am}$ Coll Cardiol 1988;11:28-34.

32 Weintraub WS, Schneider RM, Seelaus PA, Wiener DH, Agarwal JB, Helfant RH. Prospective evaluation of the severity of coronary artery disease with exercise radionuclide angiography and electrocardiography. Am Heart $\mathcal{f}$ 1986;111:537-42.

33 Detrano $R$, Gianrossi $R$, Mulvihill D, Lehmann $K$, Dubach P, Colombo A, et al. Exercise-induced ST segment depression in the diagnosis of multivessel coronary disease: a meta analysis. $\mathcal{F}$ Am Coll Cardiol 1989; 14:1501-8

34 Bonow RO, Kent KM, Rosing DR, Lan KKG, Lakotos E, Borer JS, et al. Exercise-induced ischemia in mildly symptomatic patients with coronary-artery disease and preserved left ventricular function: identification of subgroups at risk of death during medical therapy. $N$ Engl $\mathcal{F}$ Med 1984;311:1339-45.

35 Weiner DA, Ryan TJ, McCabe $\mathrm{CH}$, Chaitman BR, Sheffield LT, Fisher LD, et al. Value of exercise testing in determining the risk classification and the response to coronary artery bypass grafting in three-vessel coronary artery disease: a report from the coronary artery surgery artery disease: a report from the coronary artery surgery

36 Lee KL, Pryor DB, Pieper KS, Harrell FE Jr, Califf RM, Mark DB, et al. Prognostic value of radionuclide angiogMark DB, et al. Prognostic value of radionuclide angiography in medically treated patien

37 Millane T, Ward D. Drugs and exercise testing. BMF 1992;305:1043. 